\title{
DESIGN, SYNTHESIS AND EVALUATION OF SOME NOVEL 3-ALLYL- 6-IODO-2-SUBSTITUTED THIOQUINAZOLINONE DERIVATIVES FOR ANTICONVULSANT ACTIVITY \\ BY
}

\author{
Menshawy A. Mohamed ${ }^{a, b}$
}

\section{FROM}

${ }^{a}$ Department of Pharmaceutical Chemistry, College of Pharmacy, Salman bin Abdulaziz University, Alkharj, Saudi Arabia.

${ }^{b}$ Department of Organic Chemistry, Faculty of Pharmacy, Al-Azhar University, Cairo, Egypt.

\begin{abstract}
The urgent demand for the development of new antiepileptic drug with a better efficacy inspired us to design and synthesize a new derivatives of the fused heterocyclic analogs 3-allyl6-iodo-2-(substituted thio)quinazolin-4(3H)-one that prepared and evaluated for their anticonvulsant activity. Compounds 10, 11, 12, 13, 14 and 22 were found to be the most active anticonvulsant of this series. The achieved results proved that the distinctive compounds could be valuable as a model for future devise, acclimatization and investigation to construct more active analogues.
\end{abstract}

Key words: synthesis, 3-allyl-6-iodo-2-(substituted thio)quinazolin-4(3H)-one, anticonvulsant screening, pentylenetetrazole.

\section{Introduction}

Epilepsy is one of the most common neurological disorders, affecting about $1 \%$ of the world's population. Much efforts devoted in the recent years for the development of novel therapeutics resulted in the availability of several newer drugs as promising anticonvulsants (Stefan, H.; Feuerstein, T, 2007 , Donner, E. J.; Snead, O. C., 2006). However, the currently available anticonvulsants are effective in reducing the severity and number of seizures in less than $70 \%$ of patients. Moreover, their usage is associated with undesirable numerous side effects (Greenwood, R. S., 2000, Namara, M., et al, 2001, Löscher, W.; Schmidt, D., 2002, Bialer, M., et al, 2004).Therefore, continued search for safer and more effective anticonvulsants is urgently necessary. 
One of the most frequently encountered heterocycles in medicinal chemistry is $4(3 \mathrm{H})$ quinazolinones, which have diverse pharmacological activities such as, anticonvulsant ( Georgey H, et al, 2008, Sushil KK, et al, 2009, Hanan G, et al, 2008, Wolfe JF, et al, 1990 ) ,antimicrobial (Sami GA, 2001, Menshawy AM,et al, 2014), hypoglycemic (Mukerji DD, et al, 1985), anti-parkinsonism (Surendra SP, Shiva PS. 1979), antiviral (Pandey VK,et al, 2004), anticancer (Ashraf A. Khalil, et al,2003, Mustafa M. Ghorab ,et al, 1998), antidepressant, (Kashaw SK, et al, 2009, Wang H, et al, 2009, Jatav V, et al, 2008 ), sedative-hypnotic (Kashaw SK, et al, 2010 ) and analgesic activity (Mohsen MA, et al, 2010 ). The present study aimed to synthesize and evaluate the biological activity of some new 2-mercapto-3-allyl-4(3H)quinazolinone analogs as potential anticonvulsant. The designed target compounds were obtained as outlined in schemes 1-2 starting with 5-iodoanthranilic acid.

\section{Experimental}

All melting points shown in (table 1) were taken in open capillaries and are uncorrected. The infrared spectra were recorded on FT/IR-JASCO 4100 using $\mathrm{KBr}$ disc technique. Microanalytical data were conducted on a Perkin-Elmer 2408 analyzer, results are within $\pm 0.4 \%$ of the theoretical values, Thin layer chromatography was performed on Merk $5 \times 10 \mathrm{~cm}$ plates, percolated with silica gel GF254 using (EtOAc, hexane 1:10) as solvent system and short wavelength UV light for visualization. All fine chemicals and reagents used were purchased from Aldrich chemical Co. U.S.A. ${ }^{1} \mathrm{HNMR}$ was recorded on a Bruker $500 \mathrm{MHz}$ spectrophotometer; Chemical shifts are in $\gamma(\mathrm{ppm})$ values downfield from Tetramethyl Siloxane as an internal standard. ${ }^{13} \mathrm{C}$ NMR was recorded on a Bruker $125 \mathrm{MHz}$ spectrophotometer. The mass spectra were measured on Waters Micromass (Water AQUITY UPLC System LCT Premier XE Serial no: KE468). A preliminary screening of the anticonvulsant activity of the study compounds has indicated that some of them exhibit significant activity compared to phenobarbitone as standard drug. The starting material 2-amino-5-iodobenzoic acid (2) was synthesized in $70 \%$ yield by adapting a reported procedure (Klemme C. J. and Hunter J. H.1940).

\section{3-Allyl-2,3-dihydro-6-iodo-2-thioxoquinazolin-4(1H)-one (3)}

A mixture of 5-iodoanthranilic acid $(5.26 \mathrm{~g}, 0.02 \mathrm{~mol})$ and allylisothiocyanate $(2.3 \mathrm{ml}$, $0.02 \mathrm{~mol})$ in $30 \mathrm{ml}$ ethanol containing triethylamine $(2.0 \mathrm{~g}, 0.02 \mathrm{~mol})$ was heated under reflux for 
2 hours. The reaction mixture was poured on ice .The separated solid was filtered, washed with water, dried and crystallized from dioxane (Table 1). ${ }^{1} \mathrm{H}$ NMR (500 MHz, DMSO-d6): $\delta$ 5.0115.020 (d, 2H), 5.132-5.158 (d, 2H), 5.854-5.929(m,1H), 7.179-7.196 (dd, 1H J=8.5 Hz), 8.037$8.054(\mathrm{dd}, 1 \mathrm{H} \mathrm{J}=2$ and $8.5 \mathrm{~Hz}), 8.185(\mathrm{~d}, 1 \mathrm{H} \mathrm{J}=2.0 \mathrm{~Hz}), 13.035-13.165(\mathrm{br} \mathrm{s}, 1 \mathrm{H}) .{ }^{13} \mathrm{C}$ NMR $(125$ MHz, DMSO-d $\left.{ }_{6}\right): \delta 47.68,87.99,117.13,117.24,117.79,131.47,135.12,138.42,143.45$, 157.72, 174.89. Anal. for $\left(\mathrm{C}_{11} \mathrm{H}_{9} \mathrm{IN}_{2} \mathrm{OS}\right)$ Calcd. /Found (\%): C, 38.39 (38.46); H, 2.64 (2.45); N, 8.14 (8.14). MS: [M-1]; Calcd. /Found: 343.1705 (343.0706).

General procedure for the synthesis of 3-allyl-6-iodo-2-(alkylthio)quinazolin-4(3H)-one (414)

A mixture of compound 3-allyl-2,3-dihydro-6-iodo-2-thioxoquinazolin-4(1H)-one (3) $(0.01 \mathrm{~mol})$ and appropriate halide $(0.01 \mathrm{~mol})$ was heated under reflux for 4-6 hours in $(25 \mathrm{ml})$ acetone in the presence of anhydrous potassium carbonate $(1.0 \mathrm{~g})$. The reaction mixture was cooled; the solvent was evaporated under reduced pressure and crystallized from ethanol (Table 1).

\section{3-Allyl-6-iodo-2-(methylthio)quinazolin-4(3H)-one (4)}

${ }^{1} \mathrm{H}$ NMR (500 MHz, DMSO-d 6 ): $\delta$ 2.278(s, 3H), 4.682-4.692(d, 2H), 5.081-5.116(d, 1H, $\mathrm{J}=35 \mathrm{~Hz}), 5.190-5.211(\mathrm{~d}, 1 \mathrm{H}, \mathrm{J}=21.0 \mathrm{~Hz}), 5.861-5.937(\mathrm{~m}, 1 \mathrm{H}), 7.343-7.360$ (dd,1H, J=8.5 Hz), 8.047-8.064 (dd,1H, J=2.0 and $85 \mathrm{~Hz}), 8.313-8.317(\mathrm{~d}, 1 \mathrm{H} \mathrm{J}=2.0 \mathrm{~Hz}){ }^{13} \mathrm{C}$ NMR (125 MHz, DMSO-d 6 ) $\delta 14.52,45.89,90.27,117.40,120.39,128.07,131.09,134.57,142.98,148.08$, 158.18, 159.03. MS: [M-1; Calcd. /Found: 357.1975 (357.0787).

\section{3-Allyl-2-(ethylthio)-6-iodoquinazolin-4(3H)-one (5)}

${ }^{1} \mathrm{H}$ NMR (500 MHz, DMSO-d 6 ): $\delta$ 1.329-1.359 (t, H, $\left.\mathrm{CH}_{2} \mathrm{CH}_{3}-\mathrm{H}\right), 3.214-3.262$ (q, 2H), 4.662-4.672 (d, 2H), 5.079-5.113 (dd, 1H J=35.0), 5.185-5.205 (dd, 1H J=20.0), 5.854-5.930 (m, 1H), 7.320-7.337 (dd,1H J=8.5 Hz), 8.042- 8.059 (dd, $1 \mathrm{H} \mathrm{J}=2.0$ and $8.5 \mathrm{~Hz}), 8.311-8.315(\mathrm{~d}, 1 \mathrm{H}$ $\mathrm{J}=2.0 \mathrm{~Hz}) .{ }^{13} \mathrm{C}$ NMR $\left(125 \mathrm{MHz}, \mathrm{DMSO}-\mathrm{d}_{6}\right) \delta 13.84,26.05,45.85,90.42,117.36,120.48,128.13$, 131.17, 134.55, 142.94, 146.12, 157.55, 159.06. MS: [M-1]; Calcd. /Found: 371.2245 (371.2243). 


\section{2-(2-Hydroxyethylthio)-3-allyl-6-iodoquinazolin-4(3H)-one (6)}

${ }^{1} \mathrm{H}$ NMR (500 MHz, DMSO-d $\left.{ }_{6}\right), \delta$ 2.379(s, 1H), 3.925-4.56 (m, 4H), 5.036-5.107 (m, 2H), 5.138-5.148 (d, 2H), 5.879-5.956(m, 1H), 6.999-7.016 (d, 1H J=8.5 Hz), 7.747-7.764 (dd, $1 \mathrm{H} \mathrm{J}=2.0$ and $8.5 \mathrm{~Hz}), 8.068-8.072(\mathrm{~d}, 1 \mathrm{H} \mathrm{J}=2.0 \mathrm{~Hz}) .{ }^{13} \mathrm{C} \mathrm{NMR}\left(125 \mathrm{MHz}, \mathrm{DMSO}-\mathrm{d}_{6}\right) \delta 38.91$, 39.91, 47.70, 83.91, 115.99, 119.42, 125.28, 133.66, 134.35, 141.42, 146.90, 160.05, 175.58 . MS: [M-1]; Calcd. /Found: 387.2235 (387.4303).

\section{3-Allyl-2-(allylthio)-6-iodoquinazolin-4(3H)-one (7)}

${ }^{1} \mathrm{H}$ NMR (500 MHz, DMSO-d $\left.{ }_{6}\right)$ : $\delta$ 3.889-3.926 (d, 2H), 4.657-4.666 (d, 2H), 5.073-5.107 (dd, 1H J=34.0 Hz), 5.164-5.167 (d, 1H), 5.186-5.207 (dd,1H J=21.0), 5.849-6.026 (m, 2H),7.301-7.318 (d, 1H J=8.5 Hz), 8.013- $8.034(\mathrm{dd}, 1 \mathrm{H} \mathrm{J}=2.0$ and 8.5 Hz), 8.296-8.300 (d, 1H $\mathrm{J}=2.0 \mathrm{~Hz}) .{ }^{13} \mathrm{C}$ NMR $\left(125 \mathrm{MHz}, \mathrm{DMSO}-\mathrm{d}_{6}\right): \delta$ 34.17, 39.50, 45.89, 90.38, 119.19, 120.44, $128.15,131.10,132.59,134.56,142.98,145.86$, 156.89, 159.04. Anal. for: $\left(\mathrm{C}_{14} \mathrm{H}_{13} \mathrm{IN}_{2} \mathrm{OS}\right)$ Calcd. /Found (\%): C, 43.76 (42.61); H, 3.41 (3.18); N, 7.29 (7.05). MS: [M-1]; Calcd. /Found: $383.2355(383.2393)$.

\section{2-(3-Allyl-3,4-dihydro-6-iodo-4-oxoquinazolin-2-ylthio)acetamide (8)}

${ }^{1} \mathrm{H}$ NMR (500 MHz, DMSO-d 6 ): $\delta$ 4.477(s, 2H), 4.709-4.719 (d, 2H), 5.120-5.155 (dd,1H, J=35.0 Hz), 5.213-5.233 (dd, 1H J=20.0 Hz), 5.875-5.958 (m, 1H), 7.288 (s, 1H), 7.317$7.334(\mathrm{~d}, 1 \mathrm{H} \mathrm{J}=8.5 \mathrm{~Hz}), 7.736(\mathrm{~s}, 1 \mathrm{H}), 8.080-8.097$ (dd,1H J=2.0 and 8.5 Hz), 8.311-8.348 (d,1H $\mathrm{J}=2.0-\mathrm{Hz}) .{ }^{13} \mathrm{C}$ NMR (125 MHz, DMSO-d 6 ) $\delta$ 35.73, 46.01, 90.62, 117.58, 120.48, 128.11, 131.07, 134.89, 143.03, 146.12, 156.79, 159.03, 168.56.

\section{2-(4-Nitrobenzylthio)-3-allyl-6-iodoquinazolin-4(3H)-one (9)}

${ }^{1} \mathrm{H}$ NMR (500 MHz, $\left.\mathrm{CDCl}_{3}\right): \delta$ 4.569-4.580 (s, 2H), 4.647-4.659 (dd, 2H), 5.057-5.091 (dd, 1H), 5.179-5.200 (dd, 1H), 5.854-5.909 (m, 1H), 7.454-7.471 (d, 1H J=8.5 Hz), 7.793-7.810 $(\mathrm{dd}, 2 \mathrm{H} \mathrm{J}=5.0 \mathrm{~Hz}), 8.092-8.109(\mathrm{~d}, 1 \mathrm{H} \mathrm{J}=2.0$ and $8.5 \mathrm{~Hz}), 8.165-8.182(\mathrm{dd}, 2 \mathrm{H} \mathrm{J}=5.0 \mathrm{~Hz}), 8.321$ $(\mathrm{d}, 1 \mathrm{H} \mathrm{J}=2.0 \mathrm{~Hz}) .{ }^{13} \mathrm{C}$ NMR $\left(125 \mathrm{MHz}, \mathrm{CDCl}_{3}\right): \delta 35.89,46.42,89.64,118.91,121.08,121.66$, 127.88, 130.39, 131.01 (2), 131.75 (2), 135.40, 135.91, 143.14, 146.57, 156.46, 159.99. MS: [M1]; Calcd. /Found: 478.2925 (478.1949). 


\section{2-(4-Bromobenzylthio)-3-allyl-6-iodoquinazolin-4(3H)-one (10)}

${ }^{1} \mathrm{H}$ NMR (500 MHz, $\left.\mathrm{CDCl}_{3}\right): \delta 4.450$ (s, 2H), 4.718-4.729 (d, 2H), 5.221-5.256 (dd, 2H), 5.852-5.919 (m, 1H),7.259-7.325 (m, 4H, J=5.0 Hz), 7.430-7.447 (d, 1H, J=8.5 Hz), 7.939- 7.956 $(\mathrm{dd}, 1 \mathrm{H}, \mathrm{J}=2.0$ and $8.5 \mathrm{~Hz}), 8.535-8.538(\mathrm{~d}, 1 \mathrm{H} \mathrm{J}=2.0 \mathrm{~Hz}) .{ }^{13} \mathrm{C} \mathrm{NMR}\left(125 \mathrm{MHz}, \mathrm{CDCl}_{3}\right): \delta 35.05$, $39.51,45.89$, 90.88, 117.23, 121.53, 122.48, 124.31, 130.34, 131.71 (2), 134.33 (2), 138.63, $142.54,146.02,156.15,159.32$.

\section{2-(3-Allyl-3,4-dihydro-6-iodo-4-oxoquinazolin-2-ylthio)-N-(4-bromophenyl)acetamide (11)}

IR $\left(\mathrm{cm}^{-1}\right): 1689.34(\mathrm{C}=\mathrm{O}), 1669.34(\mathrm{C}=\mathrm{O}), 3235.97(\mathrm{NH}),{ }^{1} \mathrm{H}$ NMR (500 MHz, DMSO$\left.\mathrm{d}_{6}\right): \delta 4.195(\mathrm{~s}, 2 \mathrm{H}), 4.719-4.730(\mathrm{~d}, 2 \mathrm{H}), 5.150-5.185(\mathrm{dd}, 1 \mathrm{H}, \mathrm{J}=35.0 \mathrm{~Hz}), 5.233-5.254(\mathrm{dd}, 1 \mathrm{H}$, $\mathrm{J}=21.0 \mathrm{~Hz}), 5.896-5.972(\mathrm{~m}, 1 \mathrm{H}), 7.226-7.243$ (d,1H, J=8.5 Hz), 7.488-7.507 (dd, 2H, J=5.0 $\mathrm{Hz}), 7.567-7.587(\mathrm{dd}, 2 \mathrm{H}, \mathrm{J}=5.0 \mathrm{~Hz}), 8.052-8.069(\mathrm{dd}, 1 \mathrm{H}, \mathrm{J}=2.0$ and $8.5 \mathrm{~Hz}), 8.323-8.327$ (d, $1 \mathrm{H} \mathrm{J}=2.0 \mathrm{~Hz}$ ) 10.567 (br s, 1H). ${ }^{13} \mathrm{C}$ NMR (125 MHz, DMSO-d 6 ): $\delta 26.73,46.10,90.67,115.47$, $117.73,120.60,120.89,127.89131 .03,131.63$ (2), 134.55 (2), 143.02, 145.51, 156.02, 159.15, 166.32, 173.42. MS: [M-1]; Calcd. /Found: 553.2165 (553.2086), [M+2]: 556.2165 (556.2838).

\section{3-Allyl-2-(benzylthio)-6-iodoquinazolin-4(3H)-one (12)}

${ }^{1} \mathrm{H}$ NMR (500 MHz, DMSO-d 6 ): $\delta 4.643$ (s, 2H), 5.064-5.069 (dd, 2H), 5.164-5.184 (dd, 2H), 5.825-5.901 (m, 1H),7.254-7.269 (dd, 1H J=7.5Hz), 7.303-7.318 (dd, 1H J=7.5 Hz), 7.3807.397 (d, 1H J=8.5 Hz), 7.480-7.495 (dd, 1H J=7.5 Hz), 8.042- 8.059 (dd, 1H J=2.0 and $8.5 \mathrm{~Hz}$ ), 8.297-8.30 (d,1H J=2.0 Hz). ${ }^{13} \mathrm{C}$ NMR (125 MHz, DMSO-d 6 ): $\delta 35.58,45.90,90.44,117.51$, 120.50, 127.42, 128.08, 128.43, 129.36 (2), 131.09 (2), 136.41, 142.98, 142.54, 145.94, 157.07, 158.98. Anal. for: $\left(\mathrm{C}_{18} \mathrm{H}_{15} \mathrm{IN}_{2} \mathrm{OS}\right)$ Calcd. /Found (\%): C, 49.78 (49.56); H, 3.48 (3.64); N, 6.45 (6.44).

\section{2-(3-Allyl-3,4-dihydro-6-iodo-4-oxoquinazolin-2-ylthio)-N-phenylacetamide (13)}

${ }^{1} \mathrm{H}$ NMR (500 MHz, DMSO-d 6 ): $\delta 4.198$ (s, 2H), 4.720-4.730 (dd, 2H), 5.145-5.180 (dd, 1H), 5.232-5.252 (dd, 1H), 5.895-5.971 (m, 1H), 7.060-7.075 (dd, 1H, J=7.5 Hz), 7.258-7.276 $(\mathrm{d}, 1 \mathrm{H}, \mathrm{J}=8.5 \mathrm{~Hz}), 7.314-7.329$ (dd, 2H, J=7.5 Hz), 7.583-7.600 (dd, 2H, J=7.5 Hz), 8.054$8.071(\mathrm{dd}, 1 \mathrm{H}, \mathrm{J}=2,8.5 \mathrm{~Hz}), 8.319-8.323(\mathrm{~d}, 1 \mathrm{H}, \mathrm{J}=2.0 \mathrm{~Hz}) 10.433$ (br s, $1 \mathrm{H}) .{ }^{13} \mathrm{C}$ NMR $(125$ MHz, DMSO-d $)_{6}: \delta 36.82,46.10,90.47,117.4$ 2, 119.12, 120.47, 123.46, 127.94, 128.80, 
131.01, 134.63 (2), 138.87, 143.09, 145.92, 157.27, 157, 158.97, 165.55. MS: [M-1]; Calcd. /Found: 476.3205 (476.2409).

2-(2-(3-Allyl-3,4-dihydro-6-iodo-4-oxoquinazolin-2-ylthio)ethyl)isoindoline-1,3-dione (14)

IR $\left(\mathrm{cm}^{-1}\right): 1693.18,1708.62,1770.33(3 \mathrm{C}=\mathrm{O}) .{ }^{1} \mathrm{H}$ NMR $\left(500 \mathrm{MHz}, \mathrm{CDCl}_{3}\right): \delta 3.562-$ $3.611(\mathrm{t}, 2 \mathrm{H}), 4.131-4.18(\mathrm{t}, 2 \mathrm{H}), 4.707-4.718(\mathrm{dd}, 2 \mathrm{H}), 14.195-5.230(\mathrm{dd}, 1 \mathrm{H}), 5.213-5.233(\mathrm{dd}$, 1H), 5.828-5.905 (m, 1H), 7.366-7.383 (d, 1H, J=8.5 Hz), 7.676-7.700 (dd, 2H, J=3.0 Hz), 7.785-7.810 (dd, 2H, J= 3.0 Hz), 7.914-7.934 (dd,1H, J=2.0 and 8.5 Hz), 8.480-8.484 (d, 1H, $\mathrm{J}=2.0 \mathrm{~Hz}) .{ }^{13} \mathrm{C}$ NMR $\left(125 \mathrm{MHz}, \mathrm{CDCl}_{3}\right): \delta 30.15,36.80,46.44,89.67,118.78,119.30,120.94$, 123.32 (2), 128.33, 130.33, 131.86, 134.10 (2), 135.68, 143.06, 146.54, 156.16, 160.00, 168.08

(2). Anal. for $\left(\mathrm{C}_{21} \mathrm{H}_{16} \mathrm{IN}_{3} \mathrm{O}_{3} \mathrm{~S}\right)$ Calcd. /Found (\%): C, 48.76 (48.14); H, 3.12 (2.57); N, 8.12 (7.88). MS: [M-1]; Calcd. /Found: 516.3415 (516.1837).

\section{Ethyl 2-(3-allyl-3,4-dihydro-6-iodo-4-oxoquinazolin-2-ylthio)acetate (15)}

A mixture of compound 3-allyl-2,3-dihydro-6-iodo-2-thioxoquinazolin-4(1H)-one (3) (344 mg, 0.001mol) and ethyl 2-bromoacetate $(167 \mathrm{mg}, 0.001 \mathrm{~mol})$ was heated was heated under reflux for 4 hours in $(25 \mathrm{ml})$ acetone in the presence of anhydrous potassium carbonate $(1 \mathrm{~g})$. The reaction mixture was cooled; the solvent was evaporated under reduced pressure and crystallized from ethanol (Table 1).

IR $\left(\mathrm{cm}^{-1}\right): 1731.76,1677.77 \mathrm{~cm}^{-1}(\mathrm{C}=\mathrm{O}) .{ }^{1} \mathrm{H}$ NMR (500 MHz, DMSO-d $): \delta 1.110-1.241$ (t, 3H), 4.067-4.168 (m, 4H), 4.683-4.693 (d, 2H), 5.128-5.163 (dd, 1H, J=35.0 Hz), 5.224-5.244 (dd,1H, J=20), 5.880-5.956 (m, 1H),7.180-7.197 (d, 1H, J=8.5 Hz), 8.040- 8.057 (dd, 1H J=2.0, $8.5 \mathrm{~Hz}$ ), 8.291-8.295 (d, 1H, J=2.0 Hz). ${ }^{13} \mathrm{C}$ NMR (125 MHz, DMSO-d 6 ): $\delta$ 14.12, 34.08, 38.94, $39.61,61.10,90.60,117.66,120.36,127.85,130.91,134.62,143.09,145.75,156.79,158.82$, 168.12. Anal.for $\left(\mathrm{C}_{15} \mathrm{H}_{15} \mathrm{IN}_{2} \mathrm{O}_{3} \mathrm{~S}\right)$ Calcd. /Found (\%): C, 41.87 (42.31); H, 3.51 (3.73); N, 6.51 (6.35). MS: [M-1]; Calcd. /Found: 429.2605 (429.5111).

\section{2-(N-(2-Hydroxyethyl) acetamidothio)-3-allyl-6-iodoquinazolin-4(3H)-one (16)}

A mixture of ethyl 2-(3-allyl-3,4-dihydro-6-iodo-4-oxoquinazolin-2-ylthio)acetate (15) $(430 \mathrm{mg}, 0.001 \mathrm{~mol})$ and ethanolamine $(61 \mathrm{mg}, 0.001 \mathrm{~mol})$ in ethanol was heated under reflux for 
2 hours. The reaction mixture was cooled; the solvent was evaporated under reduced pressure and crystallized from ethanol (Table 1).

IR $\left(\mathrm{cm}^{-1}\right): 1685.18,1659.56(2 \mathrm{C}=\mathrm{O}), 3270.68(\mathrm{OH}), 3089.04(\mathrm{NH})$.

${ }^{1} \mathrm{H}$ NMR (500 MHz, DMSO-d 6 ): $\delta 1.222$ (s, 1H) 3.134-3.199 (q, 2H), 3.375-3.3.410 (q, 2H), 4.700-4.710 (d, 2H), 4.754-4.776 (d, 2H), 5.080-5.115 (dd,1H, J=35.0 Hz), 5.215-5.235 (dd, 1H J=20.0 Hz), 5.877-5.953 (m, 1H),7.335-7.352 (d,1H, J=8.5 Hz), 8.074- 8.091 (dd, 1H, $\mathrm{J}=20.0$ and $8.5 \mathrm{~Hz}), 8.322-8.326(\mathrm{~d}, 1 \mathrm{H} \mathrm{J}=2.0 \mathrm{~Hz}) 8.299(\mathrm{br} \mathrm{s}, 1 \mathrm{H}) .{ }^{13} \mathrm{C}$ NMR $(125 \mathrm{MHz}$, DMSO$\left.\mathrm{d}_{6}\right): \delta 35.75,39.61,41.84,59.60,90.43,117.60,120.45,128.13,131.04,134.56,143.03,145.97$, 157.25, 159.02, 166.64. Anal. for $\left(\mathrm{C}_{15} \mathrm{H}_{16} \mathrm{IN}_{3} \mathrm{O}_{3} \mathrm{~S}\right)$ Calcd/Found (\%): C, 40.46 (39.06); H, 3.62 (3.51); N, 9.44 (9.03). MS: [M-1]; Calcd. /Found: 444.2755: (444.2892).

\section{2-(3-Allyl-3,4-dihydro-6-iodo-4-oxoquinazolin-2-ylthio)acetohydrazide (17)}

A mixture of ethyl 2-(3-allyl-3,4-dihydro-6-iodo-4-oxoquinazolin-2-ylthio)acetate (430 mg, 0.001mol) and hydrazine hydrate $(100 \mathrm{mg}, 0.002 \mathrm{~mol})$ in ethanol was heated under reflux for 3hours. The reaction mixture was cooled; the solvent was evaporated under reduced pressure and crystallized from acetic acid (Table 1).

IR $\left(\mathrm{cm}^{-1}\right): 1662.34,1690.22(2 \mathrm{C}=\mathrm{O}), 3278.39,3056.83(\mathrm{NH})$.

${ }^{1} \mathrm{H}$ NMR (500 MHz, DMSO-d $\left.{ }_{6}\right): \delta 1.787$ (br s, 2H), 3.909 (S, 2H), 4.761-4.772 (d, 2H), 5.277-5.312 (dd, 1H, J=35.0 Hz), 5.298-5.318 (dd, 1H, J=20 Hz), 5.864-5.942 (m, 1H), 7.287$7.304(\mathrm{~d}, 1 \mathrm{H}, \mathrm{J}=8.5 \mathrm{~Hz}), 7.973-7.990(\mathrm{dd}, 1 \mathrm{H}, \mathrm{J}=2.0$ and $8.5 \mathrm{~Hz}), 8.560-8.564$ (d, 1H, J=2.0 Hz), 8.138 (br s, 1H). ${ }^{13} \mathrm{C}$ NMR (125 MHz, DMSO-d 6 ): $\delta 33.32,46.23,77.05,90.40,119.54,120.98$, 127.03, 129.97, 136.11, 143.03, 146.02, 156.98, 159.60, 169.07. MS: [M-1]; Calcd. /Found: $415.2375(415.3678)$.

\section{2-(3-Allyl-3,4-dihydro-6-iodo-4-oxoquinazolin-2-ylthio)-N-(4,5,6,7-tetrachloro-1,3- dioxoisoindolin-2-yl)acetamide(18)}

A mixture of 2-(3-allyl-3,4-dihydro-6-iodo-4-oxoquinazolin-2-ylthio)acetohydrazide (17) (416 mg $0.001 \mathrm{~mol})$ and tetrachlorophthalic anhydride $(285 \mathrm{mg}, 0.001 \mathrm{~mol})$ in glacial acetic acid $(15 \mathrm{ml})$ was heated under reflux for 18 hours. The reaction mixture was cooled; the solid obtained was filtered, washed with water, dried and crystallized from ethanol (Table 1). 
${ }^{1} \mathrm{H}$ NMR $\left(500 \mathrm{MHz}, \mathrm{CDCl}_{3}\right): \delta 4.100$ (s, 2H), 4.780-4.797 (d, 2H), 5.315-5.350 (dd, 2H, $\mathrm{J}=35 \mathrm{~Hz}), 5.889-5.966(\mathrm{~m}, 1 \mathrm{H}), 7.331-7.340(\mathrm{~d}, 1 \mathrm{H}, \mathrm{J}=8.5 \mathrm{~Hz}), 7.961-7.978(\mathrm{dd}, 1 \mathrm{H}, \mathrm{J}=2.0$ and 8.5 Hz), 8.541-8.553 (d, 1H, J=2.0 Hz), 9.909 (br s, 1H). ${ }^{13} \mathrm{C}$ NMR (125 MHz, $\left.\mathrm{CDCl}_{3}\right): \delta 33.23$, 47.00, 77.05, 90.40, 119.75, 125.68, 127.15, 129.69 (2), 130.44 (2), 136.22 (2), 140.98, 145.68, 156.87, 160.29, 164.68 (2), 169.63. MS: (m/z); [M-1]: Calcd. /Found: 683.1075 (683.1387), [M+2]: $686.1075(686.2400)$.

\section{2-(3-Allyl-3,4-dihydro-6-iodo-4-oxoquinazolin-2-ylthio)-N-(acetophenyl)acetohydrazide} (19)

A mixture of 2-(3-allyl-3,4-dihydro-6-iodo-4-oxoquinazolin-2-ylthio)acetohydrazide (17) (416 mg, 0.001 mol) and 2-phenylacetyl chloride (154 mg, 0.001mol) in N,N-dimethylformamide $(20 \mathrm{ml})$ containing pyridine $(79 \mathrm{mg}, 0.001 \mathrm{~mol})$ was heated under reflux for 20 hours. The reaction mixture was cooled; the solvent was evaporated under reduced pressure and crystallized from ethanol (Table 1).

IR $\left(\mathrm{cm}^{-1}\right):$ 1681.62, 1621.06, $1665.34\left(3 \mathrm{C}=\mathrm{O}, 3197.4,3131.55(2 \mathrm{NH}) .{ }^{1} \mathrm{H}\right.$ NMR $(500$ $\mathrm{MHz}_{\mathrm{CDCl}}$ ): $\delta 4.639$ (s, 2H), 4.960-4.970 (d, 2H), 5.293-5.313 (dd, 2H), 5.440-5.475 (dd, 2H, $\mathrm{J}=35.0 \mathrm{~Hz}$ ), 6.014-6.093 (m, 1H), 7.133 (br s, 1H), 7.169-7.184 (dd, 2H, J=7.5 Hz), 7.235 (br s, $1 \mathrm{H})$, and 7.250-7.265 (dd, 1H, J=7.5 Hz), 7.298-7.313 (dd, 2H, J=7.5 Hz),7.334-7.351 (d, 1H, $\mathrm{J}=8.5 \mathrm{~Hz}), 7.852-7.869(\mathrm{dd}, 1 \mathrm{H}, \mathrm{J}=2.08 .5 \mathrm{~Hz}), 8.662-8.666(\mathrm{~d}, 1 \mathrm{H}, \mathrm{J}=2.0 \mathrm{~Hz}) .{ }^{13} \mathrm{C}$ NMR $(125$ $\left.\mathrm{MHz}, \mathrm{CDCl}_{3}\right): \delta 34.11,45.60,77.13,90.81,117.42,118.92,119.91,127.66,127.93,128.53$, $128.73,129.34,129.52,130.15,134.02,138.34,143.20,147.38,148.63,156.79,159.19$. MS: [M-1]; Calcd. /Found: 533.3725 (533.3240)

\section{1-(2-(3-Allyl-3,4-dihydro-6-iodo-4-oxoquinazolin-2-ylthio)acetyl)-4-allylthiosemicarbazide} (20)

A mixture of compound 2- (3-ally 1-3,4-dihydro-6-iodo-4-oxoquinazolin-2-ylthio) acetohydrazide (17) (416 mg, 0.001 mol) and allylisothiocyanate (99 $\mathrm{mg}, 0.001 \mathrm{~mol})$ in dioxane was heated under reflux for 24 hours The reaction mixture was cooled;the solvent was evaporated under reduced pressure and crystallized from dioxane (Table 1). 
${ }^{1} \mathrm{H}$ NMR (500 MHz, DMSO-d 6 ): $\delta$ 4.031(s, 2H), 4.703-4.713 (dd, 2H), 4.956-4.976 (dd, $1 \mathrm{H}), 4.999-5.033$ (dd 2H), 5.118-5.153 (dd, 2H), 5.219-5.239 (dd, 2H), 5.681-5.755 (m,1H), 5.868-5.956 (m, 1H), (d, 1H, J=8.5 Hz),7.374- $7.391(\mathrm{dd}, 1 \mathrm{H} \mathrm{J}=2.0$ and $8.5 \mathrm{~Hz}), 8.292-8.308$ $(\mathrm{d}, 1 \mathrm{H} \mathrm{J}=2.0 \mathrm{~Hz}), 9.510$ (br s, 1H), 10.263 (br s, 1H). ${ }^{13} \mathrm{C}$ NMR (125 MHz, DMSO-d 6 ): $\delta 34.96$, 39.60, 45.69, 46.05, 66.33, 90.63, 115.03, 117.62, 120.44, 128.27, 130.99, 134.53, 142.96, 145.87, 157.14, 158.91, 166.61. Anal. for (\%): $\left(\mathrm{C}_{17} \mathrm{H}_{18} \mathrm{IN}_{5} \mathrm{O}_{2} \mathrm{~S}_{2}\right)$ Calcd. /Found (\%): C, 39. 62 (39.24); H, 3.52 (3.79); N, 13.59 (13.42). MS: [M-1]; Calcd. /Found: 514.3885 (514.2362).

\section{2-(3-Allyl-3,4-dihydro-6-iodo-4-oxoquinazolin-2-ylthio)-N'-tosylacetohydrazide (21)}

A mixture of 2-(3-allyl-3,4-dihydro-6-iodo-4-oxoquinazolin-2-ylthio)acetohydrazide (17) (416 mg, 0.001mol) and 4-methylbenzene-1-sulfonyl chloride (190 mg, 0.001mol) in dry pyridine $(15 \mathrm{ml})$ was heated under reflux for 24 hours. The reaction mixture was cooled and treated with ice hydrochloric acid. The separated solid was filtered, washed with water, dried and crystallized from ethanol (Table 1). ${ }^{1} \mathrm{H}$ NMR (500 MHz, $\mathrm{CDCl}_{3}$ ): $\delta 2.420$ (s, 3H), 4.573 (s, 2H), 4.754-4.764 (dd, 2H), 5.248-5.283 (dd, 1H, J=35.0 Hz), 5.310-5.330 (dd, 1H, J=20 Hz), 5.917$6.016(\mathrm{~m}, 1 \mathrm{H}),(\mathrm{dd}, 1 \mathrm{H}, \mathrm{J}=8.5 \mathrm{~Hz}), 7.747-7.348(\mathrm{dd}, 1 \mathrm{H}, \mathrm{J}=2,8.5 \mathrm{~Hz}), 7.764-7.548$ (dd, 2H, $\mathrm{J}=7.5 \mathrm{~Hz}), 8.004-8.021(\mathrm{dd}, 2 \mathrm{H}, \mathrm{J}=7.5 \mathrm{~Hz}), 8.647-8.664$ (d, $1 \mathrm{H}, \mathrm{J}=2.0 \mathrm{~Hz}), 8.740$ (br s, $1 \mathrm{H}) .{ }^{13} \mathrm{C}$ NMR (125 MHz, $\left.\mathrm{CDCl}_{3}\right): \delta$ 21.10, 39.60, 45.69, 46.05, 77.07, 90.02, 115.84, 117.02, 120.44, 127.34, 127.51, 129.50, 129.81, 131.10, 132.97, 137.18, 137.48, 146.76, 159.14, 161.91 .

\section{2-(3-Allyl-3,4-dihydro-6-iodo-4-oxoquinazolin-2-ylthio)-N-(4-methoxybenzylidene) acetohydrazide (22)}

A mixture of 2-(3-allyl-3,4-dihydro-6-iodo-4-oxoquinazolin-2-ylthio)acetohydrazide (17) (416 mg, 0.001mol) and 4-methyoxy benzaldehyde (106 mg, 0.001 mol) in glacial acetic acid (15 $\mathrm{ml}$ ) was heated under reflux for 18 hours. The reaction mixture was cooled; the obtained solid was filtered, washed with water, dried and crystallized from acetic acid (Table 1). ${ }^{1} \mathrm{H}$ NMR (500 $\left.\mathrm{MHz}, \mathrm{CDCl}_{3}\right): \delta 3.8171(\mathrm{~s}, 2 \mathrm{H}), 3.900(\mathrm{~s}, 3 \mathrm{H}), 4.992-5.003(\mathrm{~d}, 2 \mathrm{H}), 5.312-5.332(\mathrm{~d}, 1 \mathrm{H}, \mathrm{J}=20.0$ Hz), 5.471-5.506 (dd,1H, J=35.0 Hz), 6.045-6.124 (m, 1H), 6.940- 6.957 (d, 1H, J=8.5 Hz), 7.076-7.093 (dd, 2H, J=8.0 Hz), 7286-7.300 (d, 1H), 7.531-7.548 (dd, 2H, J=8.0 Hz), 7.773$7.790(\mathrm{dd}, 1 \mathrm{H}, \mathrm{J}=2.0$ and $8.5 \mathrm{~Hz}), 8.692-8.696(\mathrm{~d}, 1 \mathrm{H}, \mathrm{J}=2.0 \mathrm{~Hz}), 9.581$ (br s, $1 \mathrm{H}) .{ }^{13} \mathrm{C}$ NMR $(125$ 
$\left.\mathrm{MHz}, \mathrm{CDCl}_{3}\right): \delta 29.69,45.50,55.52,76.84,77.10,90.74,114.22,114.80,117.39,119.16$, 126.94, 130.09, 130.23, 133.27, 138.27, 142.90, 142.90, 148.27, 156.95, 161.03, 161.67. MS: [M-1]; Calcd. /Found: 533.3725 (533.6813).

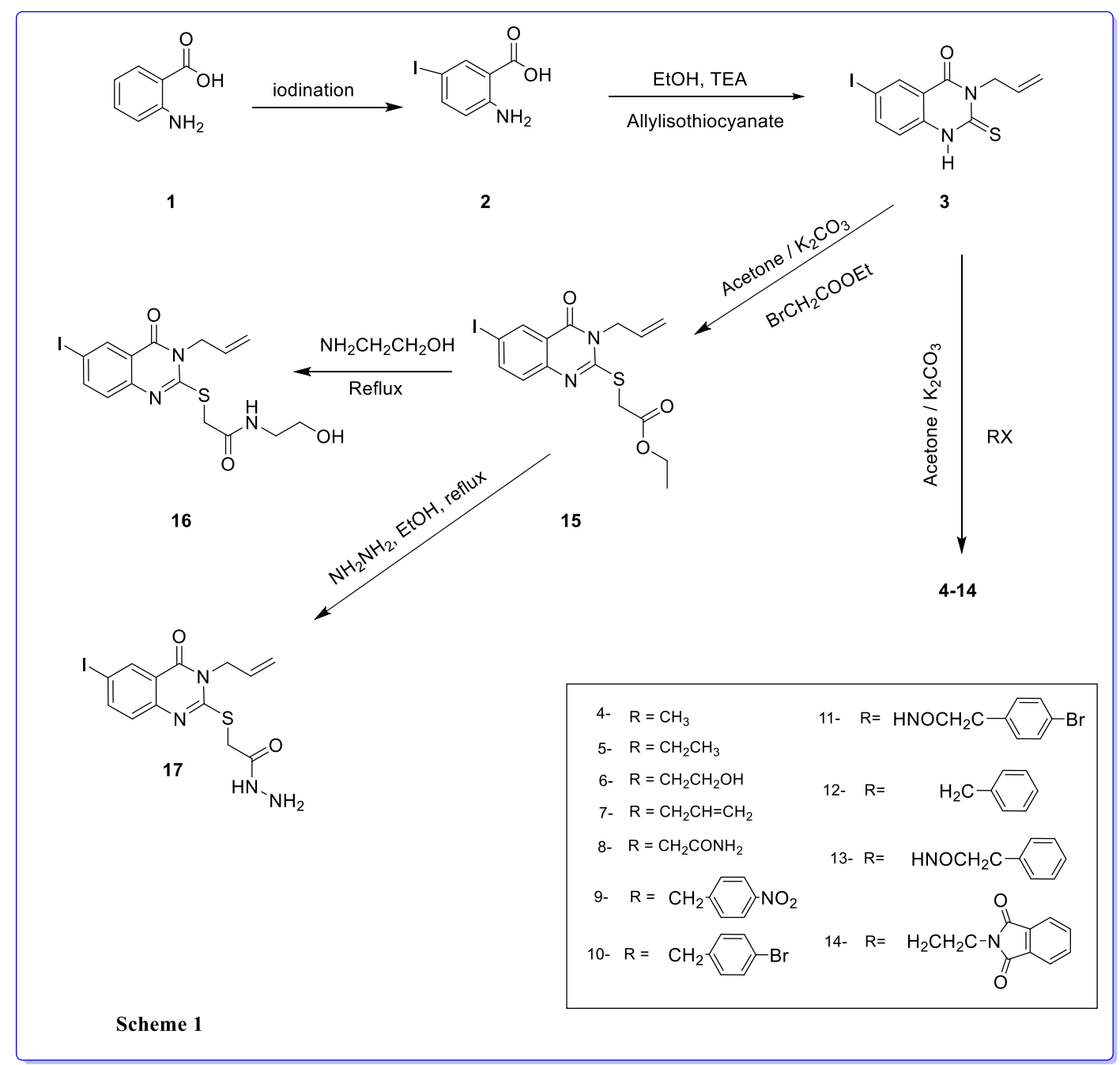




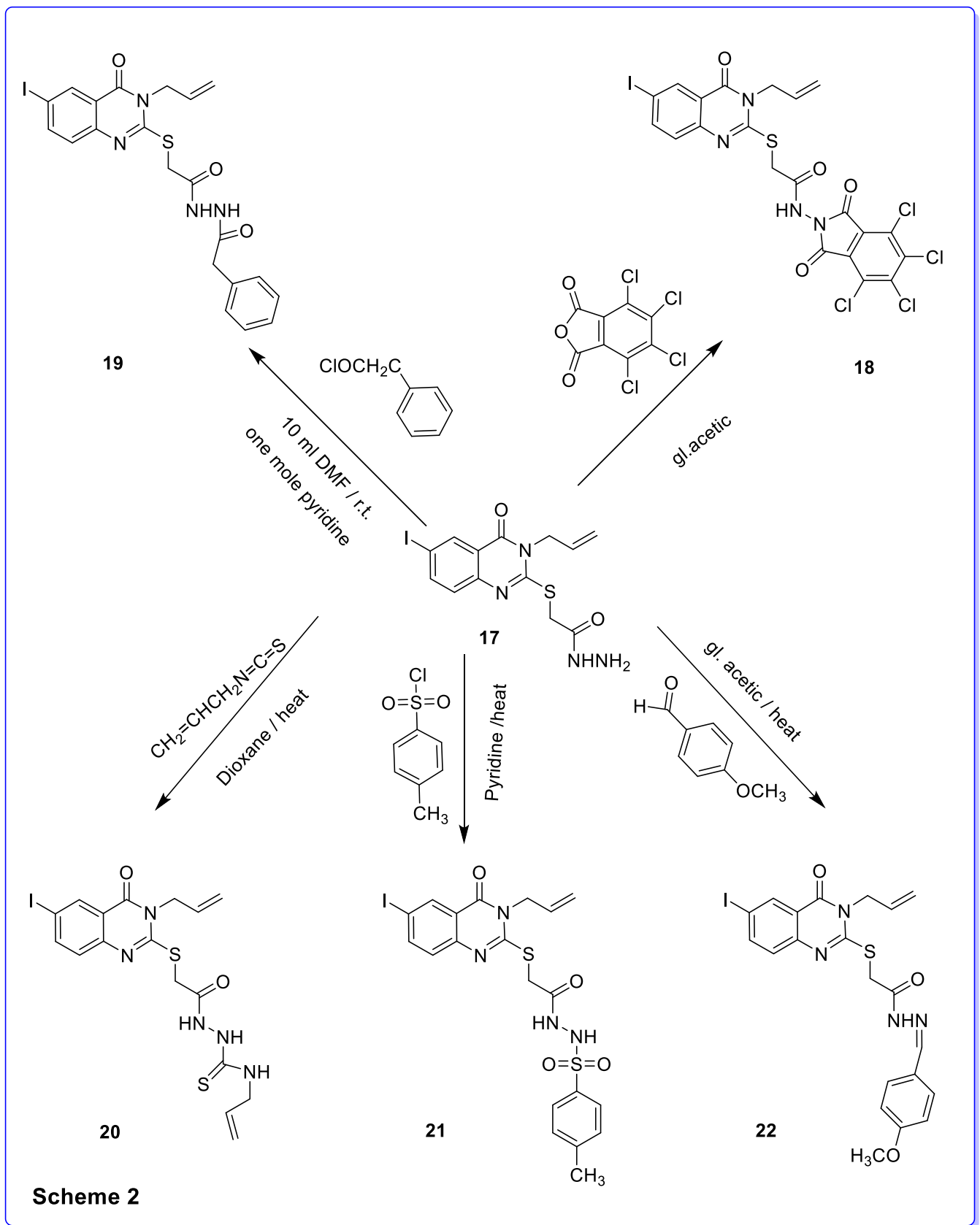


Table1: The physicochemical properties of new synthesized compo

\begin{tabular}{|c|c|c|c|c|c|}
\hline No. & Compound & $\begin{array}{c}\text { Solvent of } \\
\text { crystallization }\end{array}$ & $\begin{array}{l}\text { Melting } \\
\text { point }\end{array}$ & Yield & $\begin{array}{l}\text { Molecular } \\
\text { Formula }\end{array}$ \\
\hline 1 & 3 & Dioxane & $247-249$ & $70 \%$ & $\mathrm{C}_{11} \mathrm{H}_{9} \mathrm{IN}_{2} \mathrm{OS}$ \\
\hline 2 & 4 & Ethanol & $293-295$ & $59 \%$ & $\mathrm{C}_{12} \mathrm{H}_{11} \mathrm{IN}_{2} \mathrm{OS}$ \\
\hline 3 & 5 & Ethanol & $275-277$ & $65 \%$ & $\mathrm{C}_{13} \mathrm{H}_{13} \mathrm{IN}_{2} \mathrm{OS}$ \\
\hline 4 & 6 & Ethanol & $281-283$ & $60 \%$ & $\mathrm{C}_{13} \mathrm{H}_{13} \mathrm{IN}_{2} \mathrm{O}_{2} \mathrm{~S}$ \\
\hline 5 & 7 & Ethanol & $288-290$ & $76 \%$ & $\mathrm{C}_{14} \mathrm{H}_{13} \mathrm{IN}_{2} \mathrm{OS}$ \\
\hline 6 & 8 & Ethanol & $206-208$ & $63 \%$ & $\mathrm{C}_{13} \mathrm{H}_{12} \mathrm{IN}_{3} \mathrm{O}_{2} \mathrm{~S}$ \\
\hline 9 & 9 & Ethanol & $248-250$ & $67 \%$ & $\mathrm{C}_{18} \mathrm{H}_{14} \mathrm{IN}_{3} \mathrm{O}_{3} \mathrm{~S}$ \\
\hline 7 & 10 & Ethanol & $298-300$ & $65 \%$ & $\mathrm{C}_{18} \mathrm{H}_{17} \mathrm{BrIN}_{2} \mathrm{OS}$ \\
\hline 8 & 11 & Ethanol & $195-197$ & $72 \%$ & $\mathrm{C}_{19} \mathrm{H}_{18} \mathrm{BrIN}_{3} \mathrm{O}_{2} \mathrm{~S}$ \\
\hline 10 & 12 & Ethanol & $223-225$ & $65 \%$ & $\mathrm{C}_{18} \mathrm{H}_{17} \mathrm{IN}_{2} \mathrm{OS}$ \\
\hline 11 & 13 & Ethanol & $180-182$ & $54 \%$ & $\mathrm{C}_{19} \mathrm{H}_{18} \mathrm{IN}_{3} \mathrm{O}_{2} \mathrm{~S}$ \\
\hline 12 & 14 & Ethanol & $237-239$ & $45 \%$ & $\mathrm{C}_{21} \mathrm{H}_{16} \mathrm{IN}_{3} \mathrm{O}_{3} \mathrm{~S}$ \\
\hline 14 & 15 & Ethanol & $291-293$ & $70 \%$ & $\mathrm{C}_{15} \mathrm{H}_{15} \mathrm{IN}_{2} \mathrm{O}_{3} \mathrm{~S}$ \\
\hline 15 & 16 & Ethanol & $231-233$ & $60 \%$ & $\mathrm{C}_{15} \mathrm{H}_{16} \mathrm{IN}_{3} \mathrm{O}_{3} \mathrm{~S}$ \\
\hline 16 & 17 & Ethanol & $267-269$ & $75 \%$ & $\mathrm{C}_{13} \mathrm{H}_{13} \mathrm{IN}_{4} \mathrm{O}_{2} \mathrm{~S}$ \\
\hline 17 & 18 & Acetic acid & $228-230$ & $66 \%$ & $\mathrm{C}_{21} \mathrm{H}_{11} \mathrm{Cl}_{4} \mathrm{IN}_{4} \mathrm{O}_{4} \mathrm{~S}$ \\
\hline 18 & 19 & Ethanol & $235-237$ & $63 \%$ & $\mathrm{C}_{21} \mathrm{H}_{19} \mathrm{IN}_{4} \mathrm{O}_{3} \mathrm{~S}$ \\
\hline 19 & 20 & Dioxane & $243-244$ & $65 \%$ & $\mathrm{C}_{17} \mathrm{H}_{18} \mathrm{IN}_{5} \mathrm{O}_{2} \mathrm{~S}_{2}$ \\
\hline 20 & 21 & Ethanol & $211-213$ & $72 \%$ & $\mathrm{C}_{20} \mathrm{H}_{19} \mathrm{IN}_{4} \mathrm{O}_{4} \mathrm{~S}_{2}$ \\
\hline 21 & 22 & Acetic acid & $251-253$ & $67 \%$ & $\mathrm{C}_{21} \mathrm{H}_{19} \mathrm{IN}_{4} \mathrm{O}_{3} \mathrm{~S}$ \\
\hline
\end{tabular}




\section{Anticonvulsant activity}

\section{Materials and method}

The compounds were tested against pentylenetetrazol-induced convulsions following the method reported by (Soaje-Echaque E, and Lim RKS, 1962) using phenobarbitone sodium as reference drug. Swiss male albino mice (weigh 20-25 g) are randomly divided into 54 groups, 6 mice per each. The test 18 compounds were suspended in saline solution using few drops of tween-80 in a dose of 200, 400 and $800 \mathrm{mg} / \mathrm{kg}$. The test compounds were injected orally using same dosing volume of $0.33 \mathrm{ml}$. Phenobarbitone sodium (standard anticonvulsant), was given in doses of 6.25, 12.5 and $25 \mathrm{mg} / \mathrm{Kg}$ using the same dosing volume. Pentylenetetrazol (100 mg/Kg) was injected i.p. one hour post test compounds or standard injection. The animals were observed for 2 hours. The animal that showed no tonic convulsions within 1 hour after pentylenetetrazol injections were considered to be protected, the percentage protection was calculated per each dose, effective dose $\left(\mathrm{ED}_{50}\right)$ that protect $50 \%$ of animals against pentylentetrazol induced convulsion was then calculated using INSTAT 2 program (philadelphia). The relative potency of the test compounds to phenobarbitone sodium were calculated and used for comparison between compounds under test as shown in table 2. 
Table 2: Screening of anticonvulsant activity of the newly synthesized compounds.

\begin{tabular}{|c|c|c|c|c|c|c|}
\hline Test compound & $\begin{array}{l}\text { Dose } \\
\mathrm{mg} / \mathrm{Kg}\end{array}$ & No. of mice & $\begin{array}{l}\text { NO. of mice } \\
\text { protected }\end{array}$ & Protection \% & $\begin{array}{l}\text { Protection ED } \\
50 \% \mathrm{mg} / \mathrm{Kg}\end{array}$ & $\begin{array}{l}\text { Protection ED } \\
50 \% \mathrm{mmol} / \mathrm{Kg}\end{array}$ \\
\hline 4 & $\begin{array}{l}200 \\
400 \\
800\end{array}$ & $\begin{array}{l}6 \\
6 \\
6\end{array}$ & $\begin{array}{l}0 \\
2 \\
3\end{array}$ & 50 & 800 & 2.222 \\
\hline 5 & $\begin{array}{l}200 \\
400 \\
800\end{array}$ & $\begin{array}{l}6 \\
6 \\
6\end{array}$ & $\begin{array}{l}0 \\
2 \\
4\end{array}$ & $\begin{array}{l}33.3 \\
66.6\end{array}$ & 600 & 1.6 \\
\hline 6 & $\begin{array}{l}200 \\
400 \\
800\end{array}$ & $\begin{array}{l}6 \\
6 \\
6\end{array}$ & $\begin{array}{l}0 \\
0 \\
1\end{array}$ & 16.6 & 0 & 0 \\
\hline 8 & $\begin{array}{l}200 \\
400 \\
800\end{array}$ & $\begin{array}{l}6 \\
6 \\
6\end{array}$ & $\begin{array}{l}1 \\
3 \\
5\end{array}$ & $\begin{array}{l}50 \\
66.6\end{array}$ & 400 & 0.992 \\
\hline 9 & $\begin{array}{l}200 \\
400 \\
800\end{array}$ & $\begin{array}{l}6 \\
6 \\
6 \\
\end{array}$ & $\begin{array}{l}1 \\
3 \\
4 \\
\end{array}$ & $\begin{array}{l}16.6 \\
50 \\
66.6 \\
\end{array}$ & 400 & 0.833 \\
\hline 10 & $\begin{array}{l}200 \\
400 \\
800\end{array}$ & $\begin{array}{l}6 \\
6 \\
6\end{array}$ & $\begin{array}{l}3 \\
6 \\
6\end{array}$ & $\begin{array}{l}50 \\
100 \\
100\end{array}$ & 200 & 0.388 \\
\hline 11 & $\begin{array}{l}200 \\
400 \\
800\end{array}$ & $\begin{array}{l}6 \\
6 \\
6\end{array}$ & $\begin{array}{l}3 \\
4 \\
5\end{array}$ & $\begin{array}{l}50 \\
66.6 \\
83.3\end{array}$ & 200 & 0.358 \\
\hline 12 & $\begin{array}{l}200 \\
400 \\
800\end{array}$ & $\begin{array}{l}6 \\
6 \\
6\end{array}$ & $\begin{array}{l}3 \\
6 \\
6\end{array}$ & $\begin{array}{l}50 \\
100 \\
100\end{array}$ & 200 & 0.458 \\
\hline 13 & $\begin{array}{l}200 \\
400 \\
800\end{array}$ & $\begin{array}{l}6 \\
6 \\
6\end{array}$ & $\begin{array}{l}3 \\
4 \\
5\end{array}$ & $\begin{array}{l}50 \\
66.6 \\
83.3\end{array}$ & 200 & 0.417 \\
\hline 14 & $\begin{array}{l}200 \\
400 \\
800\end{array}$ & $\begin{array}{l}6 \\
6 \\
6\end{array}$ & $\begin{array}{l}3 \\
4 \\
4\end{array}$ & $\begin{array}{l}50 \\
66.6 \\
66.6\end{array}$ & 200 & 0.385 \\
\hline 15 & $\begin{array}{l}200 \\
400 \\
800\end{array}$ & $\begin{array}{l}6 \\
6 \\
6\end{array}$ & $\begin{array}{l}1 \\
3 \\
4\end{array}$ & \begin{tabular}{l}
\multicolumn{1}{c}{16.6} \\
50 \\
66.6
\end{tabular} & 400 & 0.930 \\
\hline 16 & $\begin{array}{l}200 \\
400 \\
800\end{array}$ & $\begin{array}{l}6 \\
6 \\
6\end{array}$ & $\begin{array}{l}0 \\
2 \\
3\end{array}$ & $\begin{array}{l}0 \\
33.3 \\
50\end{array}$ & 800 & 1.797 \\
\hline 17 & $\begin{array}{l}200 \\
400 \\
800\end{array}$ & $\begin{array}{l}6 \\
6 \\
6 \\
\end{array}$ & $\begin{array}{l}2 \\
3 \\
4 \\
\end{array}$ & $\begin{array}{l}33.3 \\
50 \\
66.6 \\
\end{array}$ & 400 & 0.961 \\
\hline 18 & $\begin{array}{l}200 \\
400 \\
800\end{array}$ & $\begin{array}{l}6 \\
6 \\
6\end{array}$ & $\begin{array}{l}2 \\
3 \\
4\end{array}$ & $\begin{array}{l}33.3 \\
50 \\
66.6\end{array}$ & 400 & 0.586 \\
\hline 19 & $\begin{array}{l}200 \\
400 \\
800\end{array}$ & $\begin{array}{l}6 \\
6 \\
6\end{array}$ & $\begin{array}{l}2 \\
3 \\
4\end{array}$ & $\begin{array}{l}33.3 \\
50 \\
66.6\end{array}$ & 400 & 0.749 \\
\hline 20 & $\begin{array}{l}200 \\
400 \\
800\end{array}$ & $\begin{array}{l}6 \\
6 \\
6\end{array}$ & $\begin{array}{l}0 \\
0 \\
0\end{array}$ & $\begin{array}{l}0 \\
0 \\
0\end{array}$ & 0 & 0 \\
\hline 21 & $\begin{array}{l}200 \\
400 \\
800\end{array}$ & $\begin{array}{l}6 \\
6 \\
6\end{array}$ & $\begin{array}{l}0 \\
2 \\
3\end{array}$ & $\begin{array}{l}0 \\
33.3 \\
50\end{array}$ & 800 & 1.403 \\
\hline 22 & $\begin{array}{l}200 \\
400 \\
800\end{array}$ & $\begin{array}{l}6 \\
6 \\
6\end{array}$ & $\begin{array}{l}33 \\
5 \\
6\end{array}$ & $\begin{array}{l}50 \\
83.3 \\
100\end{array}$ & 200 & 0.374 \\
\hline $\mathbf{S}$ & $\begin{array}{l}12.5 \\
6.5 \\
3.25\end{array}$ & $\begin{array}{l}6 \\
6 \\
6\end{array}$ & $\begin{array}{l}6 \\
3 \\
2\end{array}$ & $\begin{array}{l}100 \\
50 \\
33.3\end{array}$ & 6.5 & 0.024 \\
\hline
\end{tabular}


ED 50: median effective dose; a dose that protect $50 \%$ of animals against pentylentetrazol induced convulsion.

ED50 was calculated using INSTAT2 program (computer based statistical program)

\section{Conclusion}

The present study, new derivatives of $4(3 \mathrm{H})$-quinazolinones were synthesized and evaluated for their anticonvulsant activity in mice. The results of this study demonstrated that some 3-allyl-6-iodo-2-(substituted thio)quinazolin-4(3H)-one derivatives possess a good anticonvulsant activity, specially, compounds 10, 11, 12, 13, 14 and 22 were equipotent regarding their anticonvulsant potency as they induced 50\% protection at dose level of 200 $\mathrm{mg} / \mathrm{kg}$. Also, compounds 8, 9, 16, 17, 18 and 19 were equipotent regarding their anticonvulsant potency as they induced $50 \%$ protection at dose level of $400 \mathrm{mg} / \mathrm{kg}$. Furthermore, compounds 4 , 16 and 21 were equipotent regarding their anticonvulsant potency as they induced 50\% protection at dose level of $800 \mathrm{mg} / \mathrm{kg}$. The obtained results showed that compounds 10, 11, 12, 13, 14 and 22 could be useful as a template for future design, modification and investigation to produce more active analogs.

\section{Results and Discussion}

\section{Chemistry}

5-Iodo-2-aminobenzoic acid (2) was prepared according to a reported procedure (Klemme C. J. and Hunter J. H.1940). 3-Allyl-2, 3-dihydro-6-iodo-2-thioxoquinazolin-4(1H)one (3) as the first key intermediate was obtained in $60 \%$ yield by the reaction of 5-Iodo-2aminobenzoic acid (2) with 4-allylisothiocyanate. 2-Mercaptoquinazoline $\mathbf{3}$ was heated with different halides in dry acetone in the presence of potassium carbonate to afford the corresponding alkyl derivatives (4-15). Ethyl 2-(3-allyl-3,4-dihydro-6-iodo-4-oxoquinazolin-2ylthio)acetate (15) was refluxed with ethanolamine or hydrazine hydrate $80 \%$ in ethanol to get 2(N-(2-hydroxyethyl)acetamidothio)-3-allyl-6-iodoquinazolin-4(3H)-one (16) and 2-(3-allyl-3,4dihydro-6-iodo-4-oxoquinazolin-2-ylthio)acetohydrazide (17) as the second key intermediate (scheme 1). 2-(3-Allyl-3,4-dihydro-6-iodo-4-oxoquinazolin-2-ylthio)acetohydrazide (17) was reacted with tetrachlorophthalic anhydride and 2-phenylacetylchloride to obtain 2-(3-allyl-3,4dihydro-6-iodo-4-oxoquinazolin-2-ylthio) -N- (4,5,6,7-tetrachloro- 1 ,3 - dioxoisoindolin - 2- yl ) acetamide (18) and 2- ( 3- ally 1-3,4- dihydro -6-iodo-4-oxoquinazolin-2-ylthio)-N-(acetophenyl) 
acetohydrazide (19) in 66-63\% yield respectively. On the other hand, acid hydrazide 17 was treated with allyl isothiocyanate, 4-methylbenzenesulfonyl chloride and 4-methoxy benzaldehyde to furnish 1-(2-(3-allyl-3,4-dihydro-6-iodo-4-oxoquinazolin-2-ylthio)acetyl)-4allylthiosemicarbazide (20), (2-(3-allyl-3,4-dihydro-6-iodo-4-oxoquinazolin-2-ylthio)-N'tosylacetohydrazide (21) and 2-(3-allyl-3,4-dihydro-6-iodo-4-oxoquinazolin-2-ylthio)-N-(4 methoxybenzylidene)acetohydrazide (22) in 65,72 and $67 \%$ yield respectively (scheme 2).

\section{Anticonvulsant activity}

The anticonvulsant activities of the new synthesized compounds were evaluated by the use of standard techniques (Krall, R. L, et al, 1987, Poter, R. J, et al, 1984). The preliminary screening was performed at $200-800 \mathrm{mg} / \mathrm{kg}$ of all synthesized compounds (4-22) by using of pentylenetetrazole (PTZ) induced seizure as a chemical induction method to generate the convulsion (Vogel, H. G., 2002).

The initial anticonvulsant evaluation showed that compound $\mathbf{2 0}$ are inactive; however compounds 10, 11, 12, 13, 14 and 22 were the most active anticonvulsant agents, that caused $50 \%$ protection in a dose of $200 \mathrm{mg} / \mathrm{Kg}$ body weight while compounds $8,9,15,17,18$ and 19 were moderate activity as anticonvulsant agents caused $50 \%$ protection in a dose of $400 \mathrm{mg} / \mathrm{Kg}$, while, compound 2 exhibit $50 \%$ protection in a dose between 400 and $800 \mathrm{mg} / \mathrm{Kg}$. On the other hand, compounds 4, 16 and 21 were the least active anticonvulsant caused 50\% protection in a dose of $800 \mathrm{mg} / \mathrm{Kg}$; the compound 6 exhibited $16.6 \%$ protections at $800 \mathrm{mg} / \mathrm{Kg}$ in comparison to phenobarbitone as standard drug (table 2).

\section{Acknowledgement}

The author would like to express his special thanks to Dr. Ahmed Mansour, Department of Pharmacology, Faculty of Pharmacy, Al-Azhar University, Cairo, Egypt, for performing the Pharmacological testing of the synthesized compounds.

\section{REFERENCES}

Ashraf AK, Sami GA, and Abdulrahman MA, Hussien IE,(2003): Substituted quinazolones, part 2 invitro anticancer evaluation of new 2-substitued mercapto-3H- quinazoline analogs. Arch. Pharm. Pharm. Med. Chem., vol. 2, 95- 103. 
Bialer, M.; Johannessen, S. I.; Kupferberg, H. J.; Levy, R. H.; Perucca, E.; Tomson,(2007): T. Progress report on new antiepileptic drugs: a summary of the Eigth Eilat Conference (EILAT VIII). Epilepsy Res., 71(1), 1-52.

Donner EJ,(2006): Snead OC: New generation anticonvulsants for the treatment of epilepsy in children. NeuroRx: 3: pp 170-180

Georgey H ,Abdel-Gawad N, Abbas S.(2008): Synthesis and anticonvulsant activity of some quinazoline-4-3(H)-one derivatives. Molecules.; 13:2557-2569.

Greenwood, R.S.(2000): Adverse effects of antiepileptic drugs. Epilepsia, 41, S42.

Hanan G, Nagwa AG, Safinaz A.(2008): Synthesis and an- ticonvulsant activity of some quinazolin-4-(3H)-one. Derivatives Molecules; 13: 2557-2569.

Jatav V, Mishra P, Kashaw S, Stables JP.(2008): CNS depressant and anticonvulsant activities of some novel 3-[5-substituted 1,3,4-thiadiazole-2-yl]-2-styryl quinazoline-4(3H)-ones. Eur. J. Med. Chem.; 43:1945-1954.

Kashaw SK, Gupta V, Kashaw V, Mishra P, Stables JP, Jain NK.(2010): Anticonvulsant and sedative-hypnotic activity of some novel 3-[5-(4-substituted)phenyl-1,3,4-oxadiazole2yl]-2-styrylquinazoline-4(3H)-ones. Med. Chem. Res.; 19:250-261.

Kashaw SK, Kashaw V, Mishra P, Stables JP.(2009): Synthesis, anticonvulsant and CNS depressant activity of some new bioactive 1-(4-substituted-phenyl)-3-(4-oxo-2phenyl/ethyl-4H-quinazoline-3-yl)-urea. Eur. J. Med. Chem.; 44:4335-4343.

Klemme C. J. and Hunter J. H.,(1940): Synthesis of iodohippuric acids. 2,5-, 3,5- and 3,4diiodohippuric acids. Journal of Org. Chem., , 5, 227.

Krall, R. L.; Penry, J. K.; White, B. G.; Kupferberg, H. J.; Swinyard, E. A.(1978): Antiepileptic drug development: II. Anticonvulsant drug screening. Epilepsia., 19, 40928.

Löscher, W.; Schmidt, D.(2002): New horizons in the development of antiepileptic drugs. Epilepsy Res., 50, 3. 
Menshawy AM, Ahmed MA, Sami GA,(2014): Synthesis of some new quinazolin derivatives as potential anti-infective agents, life science journal, , 11(11), .

Mohsen MA, Yahia AM, Khairy AME, Wahid M, Basyouni SYA.(2010): Synthesis of some new 4(3H)-quina- zolinone-2-carboxaldehyde thiosemicarbazones and their metal complexes and a study on their anticonvulsant, analgesic, cytotoxic and antimicrobial activities. Eur J Med Chem; 45: 3365-3373.

Mukerji DD, Agarwal VR, NautIyal SR • (1985) : Synthesis of a new series 6 -, or 6,8 disubstituted -3-[2-substituted aniline - thiadiazolyl - 5-\{(N-mercaptoacetyl) aminomethyl\}] Quinazolin-4-ones as hypoglycemic agents. Indian J Pharm Sci; 47: 811.

Mustafa MG, Sami GA, and Sami ME.(1998): Novel quinazolone derivatives as antitumor agents. Phosphorous, Sulfur and Silicon. , vol. 142, 57- 68.

Namara, M.; Hardman, G. J.; Limbird, L. E.; Gilman, A. G.(2001): Drugs Effective in the Therapy of the Epilepsies; McGraw-Hill: New York,. pp 521-548.

Pandey VK, Sarah T, Zehra T.(2004): Thiadiazolyl quinazolones as potential anti-viral agents, Indian J Chem; 43: 180-183.

Poter, R. J.; Cereghino, J. J.; Gladding, G. D.; Hessie, B. J.; Kupferberg, H. J.;Scoville, B. (1984):Antiepileptic Drug Development Program. Cleveland Clin., Q 51, 293-305.

Sami GA,(2001): Synthesis of some new substituted quinazoline derivatives and their antimicrobial screening. Saudi Pharmaceutical Journal, vol. 9, No 2, 72- 83.

Soaje - Echaque E, Lim RKS (1962): Anticonvulsant activity of some carbonyl ureas. J. Pharmacol Exp. Ther. 138:224-228

Surendra SP, Shiva PS.( 1979): Synthesis of substituted 2- methyl-3-(3,4-dimethoxy/ dihydroxyphenylethyl)-4- quinazolones as possible Antiparkinson drugs. J Heterocyclic Chem; 16: 449-452. 
Sushil KK, Varsha K, Pradeep M, Jain NK, Stables JP.( 2009): Synthesis, anticonvulsant and CNS depressant activity of some new bioactive 1-(4-substituted-phenyl)-3-(4-oxo-2phenyl/ethyl-4H- quinazolin-3-yl)urea. Eur J Med Chem; 44: 4335-4343.

Stefan, H.; Feuerstein, T. (2007):Novel anticonvulsant drugs. Pharmacol. Ther., 113,165.

Vogel, H. G. (2002):In Drug Discovery and Evaluation; Vogel, H. G., Vogel, W. H., Eds., 2nd ed.; Pharmacological assays; Springer: Berlin,; pp. 422-488.

Wang H, Wei C, Deng X, Li F, Quan Z. (2009):Synthesis and evaluation on anticonvulsant and antidepressant activities of 5-Alkoxy-tetrazolo[1,5-a]quinazolines. Arch. Pharm. Chem. Life Sci.; 342:671-675.

Wolfe JF, Rathman TL, Sleevi MC, Campbell JA, Greenwood TD. (1990):Synthesis and anticonvulsant activity of some new 2-subtituted 3-aryl-4(3H)- quinazolinones. J Med Chem; 33: 161-166.

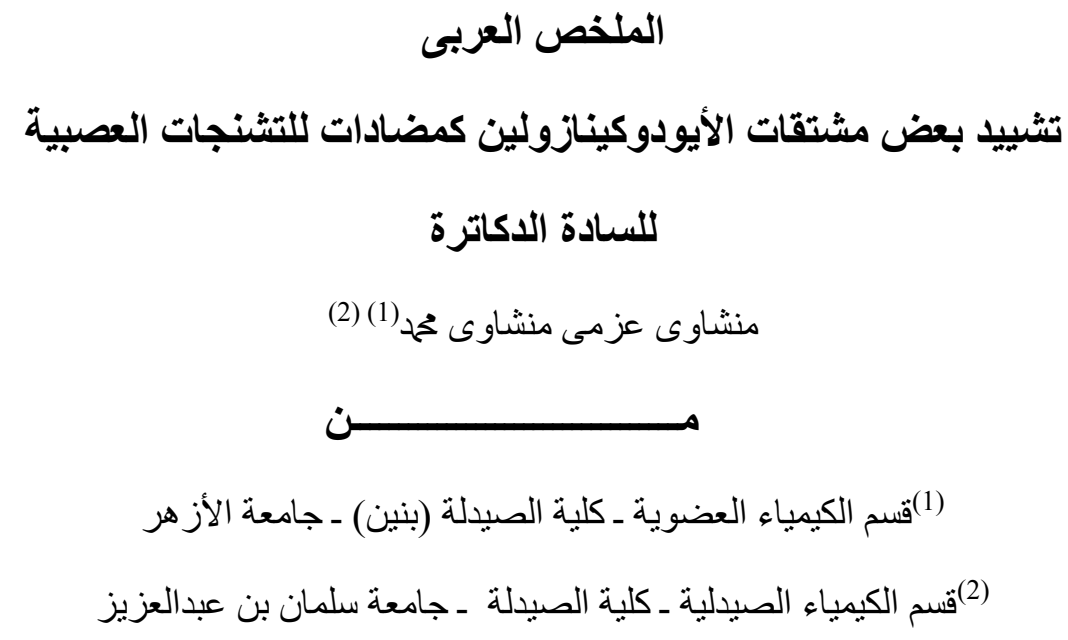

فى هذا البحث تم تتبيد عدد 20 مركبا وسيطا ونهائيا من نواة الأيودوالكينازولين وقد تم إثباتها بطرق التحاليل المختلفة كنقطة الأنصهاروالرنين النووى المغناطيسى ومطياف الكتلة والتحليل الدقيق للعناصر(الكربون و النيتروجين و الهيدروجين) لبعض المركبات المشيدة وتم إختبار ها كمضادات للتشنجات مقارنة بالفينوباربيتون صوديو و وقد ثبت للمركبات الجديدة فاعلية كمضادات للتشنجات وخاصة المركبات أرقام 10و 11و12و13و14و التى ثبت لها فاعلية فى عمل 50\% 